\title{
Early versus late Gamma Knife radiosurgery for Cushing's disease after prior resection: results of an international, multicenter study
}

\author{
Adomas Bunevicius, MD, PhD, ${ }^{1}$ Hideyuki Kano, MD, PhD, ${ }^{2}$ Cheng-Chia Lee, MD, PhD, ${ }^{3}$ \\ Michal Krsek, MD, ${ }^{4}$ Ahmed M. Nabeel, MD, PhD, ${ }^{5}$ Amr El-Shehaby, MD, PhD, ${ }^{6}$ \\ Khaled Abdel Karim, MD, PhD, ${ }^{7}$ Nuria Martinez-Moreno, MD, PhD, ${ }^{8}$ David Mathieu, MD, ${ }^{9}$ \\ John Y. K. Lee, MD, MSCE, ${ }^{10}$ Inga Grills, MD,,1 Douglas Kondziolka, MD,12 \\ Roberto Martinez-Alvarez, MD, PhD, ${ }^{8}$ Wael A. Reda, MD, PhD, ${ }^{6}$ Roman Liscak, MD, PhD, ${ }^{13}$ \\ Yan-Hua Su, MD, ${ }^{3}$ L. Dade Lunsford, MD, ${ }^{2}$ Mary Lee Vance, MD, ${ }^{1,14}$ and \\ Jason P. Sheehan, MD, PhD'
}

1Department of Neurologic Surgery, University of Virginia Health System, Charlottesville, Virginia; ${ }^{2}$ Department of Neurologic Surgery, University of Pittsburgh Medical Center, Pittsburgh, Pennsylvania; ${ }^{3}$ Department of Neurosurgery, Neurologic Institute, Taipei Veterans General Hospital, Taipei, Taiwan, Republic of China; ${ }^{2}$ Third Department of Internal Medicine, First Faculty

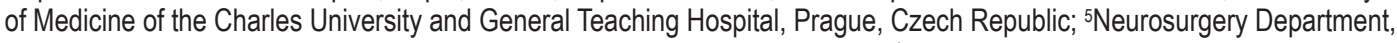
Gamma Knife Center Cairo-Nasser Institute, Benha University, Benha, Egypt; ${ }^{6}$ Neurosurgery Department, Gamma Knife Center Cairo-Nasser Institute, Ain Shams University, Cairo, Egypt; ${ }^{7}$ Clinical Oncology Department, Gamma Knife Center Cairo-Nasser Institute, Ain Shams University, Cairo, Egypt; ${ }^{8}$ Department of Functional Neurosurgery and Radiosurgery, Ruber International Hospital, Madrid, Spain; ' ${ }^{D}$ ivision of Neurosurgery, Université de Sherbrooke, Centre de recherche du CHUS, Sherbrooke, Quebec, Canada; ${ }^{10}$ Department of Neurosurgery, University of Pennsylvania, Philadelphia, Pennsylvania; ${ }^{11}$ Department of Radiation Oncology, Beaumont Health System, Royal Oak, Michigan; ${ }^{2}$ Department of Neurosurgery, New York University Langone Medical Center, New York, New York; ${ }^{13}$ Department of Stereotactic and Radiation Neurosurgery, Na Homolce Hospital, Prague, Czech Republic; and ${ }^{14}$ Department of Medicine, University of Virginia Health System, Charlottesville, Virginia

OBJECTIVE The optimal time to perform stereotactic radiosurgery after incomplete resection of adrenocorticotropic hormone (ACTH)-producing pituitary adenoma in patients with Cushing's disease (CD) remains unclear. In patients with persistent $\mathrm{CD}$ after resection of $\mathrm{ACTH}$-producing pituitary adenoma, the authors evaluated the association of the interval between resection and Gamma Knife radiosurgery (GKRS) with outcomes.

METHODS Pooled data from 10 institutions participating in the International Radiosurgery Research Foundation were used in this study.

RESULTS Data from 255 patients with a mean follow-up of $65.59 \pm 49.01$ months (mean \pm SD) were analyzed. Seventy-seven patients (30\%) underwent GKRS within 3 months; 46 (18\%) from 4 to 6 months; 34 (13\%) from 7 to 12 months; and $98(38 \%)$ at $>12$ months after the resection. Actuarial endocrine remission rates were higher in patients who underwent GKRS $\leq 3$ months than when treatment was $>3$ months after the resection $(78 \%$ and $65 \%$, respectively; $p=0.017)$. Endocrine remission rates were lower in patients who underwent GKRS at $>12$ months versus $\leq 12$ months after the resection ( $57 \%$ vs $76 \%$, respectively; $p=0.006$ ). In multivariate Cox regression analyses adjusted for clinical and treatment characteristics, early GKRS was associated with increased probability of endocrine remission (hazard ratio $[H R] 1.518,95 \% \mathrm{Cl} 1.039-2.218 ; p=0.031)$, whereas late GKRS (HR 0.641, 95\% Cl 0.448-0.919; $p=0.015)$ was associated with reduced probability of endocrine remission. The incidence of some degree of new pituitary deficiency ( $p$ $=0.922)$, new visual deficits $(p=0.740)$, and other cranial nerve deficits $(p=0.610)$ was not significantly related to time from resection to GKRS.

ABBREVIATIONS $\mathrm{ACTH}=$ adrenocorticotropic hormone; $\mathrm{CD}=$ Cushing's disease; CKSRS = CyberKnife SRS; GKRS = Gamma Knife radiosurgery; $\mathrm{HR}=$ hazard ratio; LINAC = linear accelerator; SRS = stereotactic radiosurgery; UFC = urinary free cortisol.

SUBMITTED October 17, 2019. ACCEPTED December 13, 2019.

INCLUDE WHEN CITING Published online February 21, 2021; DOI: 10.3171/2019.12.JNS192836. 
CONCLUSIONS Early GKRS is associated with an improved endocrine remission rate, whereas later GKRS is associated with a lower rate of endocrine remission after pituitary adenoma resection. Early GKRS should be considered for patients with CD after incomplete pituitary adenoma resection.

https://thejns.org/doi/abs/10.3171/2019.12.JNS192836

KEYWORDS Cushing's disease; stereotactic radiosurgery; remission; pituitary surgery

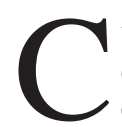
USHING's disease $(\mathrm{CD})$ is caused by an adrenocorticotropic hormone (ACTH)-secreting pituitary adenoma resulting in uncontrolled cortisol hypersecretion by the adrenal glands. Persistent hypercortisolemia is associated with increased risk for numerous somatic and psychiatric morbidities, including central obesity, diabetes, hypertension, mood changes, and accelerated cognitive decline.., 21

Furthermore, patients with CD have up to a 5-fold increased mortality risk when compared with the general population. ${ }^{7,9}$ The greater morbidity and mortality risk of patients with $\mathrm{CD}$ is associated with greater duration of hypercortisolemia, ${ }^{8}$ lower rate of endocrine remission after pituitary adenoma resection (i.e., failed pituitary surgery), ${ }^{4}$ and higher number of prior treatments. ${ }^{2}$ Therefore, timely and adequate control of cortisol hypersecretion is important for a favorable prognosis in these patients.

The first-line treatment for patients with established $\mathrm{CD}$ is resection of the ACTH-producing pituitary adenoma, and this is usually accomplished via a transsphenoidal route. ${ }^{15,16,23}$ However, up to $35 \%$ of patients with CD do not achieve endocrine remission after pituitary surgery, and an additional significant proportion of patients experience tumor progression and/or recurrence of hypercortisolemia. ${ }^{18,19}$ Persistent hypercortisolemia after initial adenoma resection can sometimes be successfully managed with lifelong medical therapy ${ }^{20}{ }^{2}$ However, lifelong medical treatment for hypercortisolemia can be expensive, places patients at increased risk for treatment-related adverse events, and does not cure CD. ${ }^{20}$ Repeated resection of residual and/or recurrent ACTH-producing pituitary adenomas is sometimes attempted; however, it has a lower success rate and greater complication risk when compared with initial surgery. ${ }^{1,10,17}$ Consequently, stereotactic radiosurgery (SRS) is currently recommended for the management of patients with $C D$ who have persistent hypercortisolemia and/or post-SRS tumor recurrence. ${ }^{15}$ SRS has an acceptable safety profile and often leads to durable endocrine remission in up to $80 \%$ of patients..$^{13}$ However, there are no large studies evaluating the optimal time from adenoma resection to SRS. This knowledge may have important clinical implications for treatment guidance after incomplete resection or after disease recurrence.

From a large consecutive sample of patients with established $\mathrm{CD}$, we investigated the possible association of the interval between the resection of ACTH-producing pituitary adenoma and SRS with the treatment outcomes reported.

\section{Methods \\ Patient Population}

Patients were identified from institutions affiliated with the International Radiosurgery Research Foundation (protocol R-16-10) who were treated with Gamma Knife radiosurgery (GKRS) between 1990 and 2014. Data collection was approved by institutional review boards at each of the participating centers. A database with variables of interest was established by investigators at the University of Virginia and sent to all participating centers. Individual patient data were de-identified and pooled for the analyses. For the purpose of this study, we considered only patients who were diagnosed with $\mathrm{CD}$ according to the existing guidelines and who underwent pituitary adenoma resection before GKRS, via either transsphenoidal approach or craniotomy. Patients who did not have previous surgery for a pituitary adenoma were excluded. Also, patients were required to have at least one follow-up evaluation including endocrine testing and neuroimaging testing after GKRS to be included in the study. In the delayed postoperative GKRS group, all patients had documented endocrine evidence of active $\mathrm{CD}$. In the early postoperative GKRS cohort, patients had active CD reflected by the lack of a serum cortisol nadir after resection, elevated cortisol concentration, and/or persistent CD symptoms.

As described previously, the diagnosis of $\mathrm{CD}$ was established according to existing guidelines ${ }^{15}$ and included testing of serum cortisol, ACTH, 24-hour urinary free cortisol (UFC), and other tests as appropriate. ${ }^{13}$

\section{Radiosurgical Approach}

Single-session GKRS was performed using models U, B, C, 4C, and Perfexion of Gamma Knife units (Elekta $\mathrm{AB})$. Frame-based stereotaxy was performed using the Leksell model $G$ frame (Elekta $A B$ ), which was placed under local anesthesia with or without conscious sedation. Imaging studies for radiosurgical planning typically included high-resolution 1-mm thickness pre- and postcontrast T1-weighted MRI scans. In rare cases in which MRI was contraindicated, stereotactic CT was used for GKRS planning. GKRS dose planning was performed by a multidisciplinary team that included a neurosurgeon, radiation oncologist, and medical physicist. At each center, planning was tailored toward individual patient needs and imaging findings. However, the maximal radiation dose to the optic nerve, chiasms, and tracts was generally kept below 8-12 Gy. Radiosurgical parameters, including the margin and maximum dose, optic apparatus maximal dose, isodose line, tumor volume, and number of isocenters, were recorded.

\section{Clinical and Radiological Follow-Up}

The interval from the most recent pituitary adenoma operation until initial GKRS was recorded in all patients. Although clinical and endocrine follow-up varied across 
practices, follow-up typically included 24-hour UFC, and MRI at 6-month intervals for the first 2 years and annually thereafter. Endocrine remission was defined as normalization of UFC or morning serum cortisol (as determined by institutional reference ranges) with the patient off of medication to control hypercortisolism. The interval from GKRS to endocrine remission was recorded in all patients. Endocrine recurrence was defined as increase of 24-hour UFC or serum morning cortisol concentration above institutional reference ranges. Tumor progression or response was defined as an increase of tumor volume by $20 \%$ or a decrease by $20 \%$, respectively. Adverse radiation events and other treatments were carefully monitored.

\section{Statistical Analyses}

Statistical analyses were performed with IBM SPSS Statistics for Windows version 25.0 (IBM Corp.). All identified patients were stratified into 4 cohorts based on the interval between surgical tumor resection and GKRS: 1) $\leq$ 3 months; 2) from 4 to 6 months; 3) from 7 to 12 months; and 4) later than 12 months. Categorical data were compared using the Pearson chi-square test, and continuous data using the ANOVA. Kaplan-Meier analysis with log-rank test was performed to analyze the possible association of endocrine remission rate with interval from tumor surgery until GKRS. Next, univariate Cox regression analysis was carried out to evaluate the association between the prespecified intervals from tumor surgery to GKRS ( $\leq 3$ months vs $>3$ months and $\leq 12$ months vs $>12$ months) and endocrine remission rates at 6 months, 1 year, 2 years, 5 years, and at later times after SRS. Significant associations in univariate Cox regression analyses were then adjusted for patient age, sex, pre-GKRS fractionated radiation therapy, medical treatment at time of GKRS, pre-GKRS UFC concentration, volume treated, maximal GKRS dose (Gy), margin dose (Gy), indication for GKRS (residual or recurrent disease), and treatment of the whole sella and cavernous sinuses.

\section{Results}

\section{Patient Characteristics}

Two hundred fifty-five patients with CD who underwent GKRS after resection of an adenoma were identified by 10 participating institutions. The mean age of the study patients at the time of GKRS was $41.36 \pm 13.54$ years (mean $\pm \mathrm{SD}$, range $10-81$ years), and the majority $(79 \%)$ of patients were women (Table 1). Seventy-seven (30\%) patients underwent GKRS within 3 months after resection; 46 (18\%) from 4 to 6 months after resection; 34 (13\%) from 7 to 12 months after resection; and $98(38 \%)$ at more than 12 months after surgery. Patients who underwent GKRS at more than 12 months after prior resection were more often subjected to GKRS for recurrent adenoma ( $p$ $<0.001$ ) and had a greater prevalence of pre-GKRS radiation therapy for their adenomas $(\mathrm{p}=0.015$; Table 1$)$. $\mathrm{Pa}-$ tients who underwent GKRS within 3 months of surgery had a lower prevalence of a history of medical treatment $(p=0.021)$ and were less likely to use medical therapy at the time of GKRS ( $p<0.001)$. Distribution of age, sex, histories of surgery, prevalence of pre-GKRS pituitary defi- ciency, visual deficits, and concentrations of 24-hour UFC and morning serum cortisol were similar as a function of the interval between surgery and GKRS.

\section{Radiosurgical Parameters}

Patients treated with GKRS within 3 months after surgery were more likely to have GKRS to the cavernous sinus or sinuses $(\mathrm{p}<0.002)$, and their isodose line $(\%)$ was lower relative to other groups of patients $(\mathrm{p}<0.001$; Table 2 ). Other GKRS characteristics were similar among the 4 groups of patients stratified according to GKRS timing.

\section{Endocrine Remission}

Following GKRS, the mean endocrine follow-up in the entire cohort of patients was $65.59 \pm 49.01$ months (median 51 months), and it was similar among the 4 groups of patients stratified according to GKRS timing ( $\mathrm{p}=0.382$; Table 1). In the total sample of patients, actuarial endocrine remission rates at 1,2 , and 5 years and at last followup after GKRS were 43\%, 57\%, 68\%, and 69\%, respectively. In patients treated with GKRS within 3 months of resection, remission rates at 1,2 , and 5 years and at last follow-up after GKRS were 52\%, 70\%, 77\%, and 78\%, respectively. Endocrine remission rates at 1, 2, and 5 years and at last follow-up were 48\%, 64\%, 75\%, and 76\%, respectively, among patients treated with GKRS at $\leq 12$ months after the resection, and $36 \%, 45 \%, 56 \%$, and $69 \%$, respectively, among patients treated with GKRS at $>12$ months after the resection. In the total sample of patients, there was a significant association of time interval from surgery to GKRS ( $\leq 3$ months vs from 4 to 6 months vs from 7 to 12 months vs later than 12 months) with actuarial endocrine remission rates at last follow-up visit (logrank test $=12.644, p=0.005)$. Specifically, Kaplan-Meier analyses showed that when compared with CD patients who were treated with GKRS $>3$ months after resection of the adenoma, patients who were treated with GKRS $\leq$ 3 months after resection had higher actuarial endocrine remission rates at 6 months (20\% vs 33\%, respectively); at 2 years (51\% vs 70\%, respectively); and at last followup $(65 \%$ vs $78 \%$, respectively; log-rank test $=5.716, p=$ 0.017; Fig. 1 upper). On the other hand, in patients with $C D$ who were treated with GKRS $>12$ months after resection when compared to those who were treated with GKRS $\leq$ 12 months after pituitary adenoma resection, actuarial endocrine remission rates were lower at 6 months $(25 \%$ vs $21 \%$, respectively); at 2 years (64\% vs $45 \%$, respectively); and at last follow-up (76\% vs 57\%, respectively; log-rank test $=7.457, \mathrm{p}=0.006$; Fig. 1 lower $)$.

In univariate Cox regression analyses an interval between prior resection and an initial GKRS of $\leq 3$ months was associated with statistically significantly greater hazard ratios (HRs) for endocrine remission at 6 months, 2 years, and last follow-up after GKRS, whereas an interval from resection to GKRS of $>12$ months was associated with lower likelihood for endocrine remission at 2 years and last follow-up after the GKRS (Table 3). After adjusting for patient age, sex, pre-GKRS fractionated radiation therapy, medical treatment at time of GKRS, pre-GKRS UFC concentration, volume treated, maximal GKRS dose 
TABLE 1. Baseline clinical and demographic characteristics as a function of time between resection and radiosurgery in 255 patients with CD

\begin{tabular}{|c|c|c|c|c|c|c|}
\hline \multirow[b]{2}{*}{ Characteristic } & \multirow[b]{2}{*}{ Total Sample } & \multicolumn{4}{|c|}{ Time Interval Between Resection and Radiosurgery } & \multirow[b]{2}{*}{$\mathrm{p}$ Value } \\
\hline & & $\leq 3 \mathrm{Mos}$ & 4-6 Mos & $7-12$ Mos & $>12$ Mos & \\
\hline No. of patients & 255 & $77(30 \%)$ & $46(18 \%)$ & $34(13 \%)$ & $98(38 \%)$ & \\
\hline Sex & & & & & & $1.691(0.639)$ \\
\hline Men & $54(21 \%)$ & $19(25 \%)$ & $7(15 \%)$ & $8(23 \%)$ & $20(20 \%)$ & \\
\hline Women & $201(79 \%)$ & $58(75 \%)$ & $39(85 \%)$ & $26(77 \%)$ & $78(80 \%)$ & \\
\hline Age at GKRS, yrs & $41.36 \pm 13.54$ & $41.57 \pm 12.27$ & $40.39 \pm 14.76$ & $43.82 \pm 15.25$ & $40.82 \pm 13.38$ & $0.511(0.675)$ \\
\hline Radiosurgery indications & & & & & & $28.199(<0.001)$ \\
\hline Residual & $221(87 \%)$ & $76(99 \%)$ & $42(91 \%)$ & $31(91 \%)$ & $72(73 \%)$ & \\
\hline Recurrent & $34(13 \%)$ & $1(1 \%)$ & $4(9 \%)$ & $3(9 \%)$ & $26(27 \%)$ & \\
\hline Pre-GKRS transsphenoidal resection & & & & & & $4.274(0.233)$ \\
\hline Yes & $248(97 \%)$ & $77(100 \%)$ & $45(98 \%)$ & $33(97 \%)$ & $93(95 \%)$ & \\
\hline No & $7(3 \%)$ & $0(0 \%)$ & $1(2 \%)$ & $1(3 \%)$ & $5(5 \%)$ & \\
\hline Pre-GKRS craniotomy & & & & & & $4.726(0.193)$ \\
\hline Yes & $11(4 \%)$ & $2(3 \%)$ & $0(0 \%)$ & $2(6 \%)$ & $7(7 \%)$ & \\
\hline No & $244(96 \%)$ & $75(97 \%)$ & $46(100 \%)$ & $32(94 \%)$ & $91(93 \%)$ & \\
\hline Pre-GKRS fractionated radiation therapy & & & & & & $10.403(0.015)$ \\
\hline Yes & $16(6 \%)$ & $3(4 \%)$ & $0(0 \%)$ & $1(3 \%)$ & $12(12 \%)$ & \\
\hline No & $239(94 \%)$ & $74(96 \%)$ & $46(100 \%)$ & $33(97 \%)$ & $86(88 \%)$ & \\
\hline Pre-GKRS medical therapy & & & & & & $9.726(\mathbf{0 . 0 2 1})$ \\
\hline Yes & $120(47 \%)$ & $25(33 \%)$ & $24(52 \%)$ & $17(50 \%)$ & $54(55 \%)$ & \\
\hline No & $135(53 \%)$ & $52(67 \%)$ & $22(48 \%)$ & $17(50 \%)$ & $44(45 \%)$ & \\
\hline Medical therapy at time of GKRS & & & & & & $25.589(<0.001)$ \\
\hline Yes & $83(32 \%)$ & $8(10 \%)$ & $17(37 \%)$ & $14(41 \%)$ & $44(45 \%)$ & \\
\hline No & $172(68 \%)$ & $69(90 \%)$ & $29(63 \%)$ & $20(58 \%)$ & $54(55 \%)$ & \\
\hline \multicolumn{7}{|l|}{ Pituitary deficiency before GKRS* } \\
\hline Any & $70(28 \%)$ & $23(30 \%)$ & $10(23 \%)$ & $10(30 \%)$ & $27(28 \%)$ & $0.890(0.828)$ \\
\hline Hypothyroidism & $50(20 \%)$ & $18(24 \%)$ & $5(11 \%)$ & $9(27 \%)$ & $18(19 \%)$ & $3.786(0.286)$ \\
\hline Estrogen/testosterone deficiency & $18(7 \%)$ & $7(9 \%)$ & $3(7 \%)$ & $2(6 \%)$ & $6(6 \%)$ & $0.618(0.892)$ \\
\hline GH deficiency & $8(3 \%)$ & $3(4 \%)$ & $1(2 \%)$ & $2(6 \%)$ & $2(2 \%)$ & $1.462(0.691)$ \\
\hline Diabetes insipidus & $13(5 \%)$ & $5(7 \%)$ & $2(5 \%)$ & $2(6 \%)$ & $4(4 \%)$ & $0.543(0.909)$ \\
\hline Visual deficits before GKRS & & & & & & $3.505(0.743)$ \\
\hline None & $220(86 \%)$ & $68(88 \%)$ & $41(89 \%)$ & $29(85 \%)$ & $82(84 \%)$ & \\
\hline Visual field deficit & $25(10 \%)$ & $5(7 \%)$ & $4(9 \%)$ & $3(9 \%)$ & $13(13 \%)$ & \\
\hline Diplopia & $10(4 \%)$ & $4(5 \%)$ & $1(2 \%)$ & $2(6 \%)$ & $3(3 \%)$ & \\
\hline Pretreatment 24-hr UFC, $\mu \mathrm{g} \dagger$ & $278.2 \pm 490.4$ & $181.4 \pm 181.9$ & $384.2 \pm 839.3$ & $209.1 \pm 193.1$ & $320.6 \pm 488.2$ & $1.758(0.156)$ \\
\hline Pretreatment morning serum cortisol, $\mu \mathrm{g} / \mathrm{dL} \ddagger$ & $25.5 \pm 19.6$ & $24.0 \pm 16.1$ & $26.0 \pm 21.0$ & $25.6 \pm 24.9$ & $26.5 \pm 19.6$ & $0.215(0.886)$ \\
\hline Time between resection and GKRS, mos & $23.17 \pm 37.19$ & $2.18 \pm 0.68$ & $5.09 \pm 0.86$ & $8.88 \pm 1.87$ & $53.10 \pm 49.23$ & $57.958(<0.001)$ \\
\hline Endocrine follow-up, mos & $65.59 \pm 49.01$ & $62.57 \pm 44.70$ & $70.79 \pm 52.96$ & $76.42 \pm 54.34$ & $61.75 \pm 48.36$ & $1.024(0.382)$ \\
\hline
\end{tabular}

$\mathrm{GH}=$ growth hormone.

Values are expressed as the mean \pm SD or the number (\%). The p values (in parentheses) were calculated as follows: categorical data were compared using the Pearson chi-square test, and continuous data using the ANOVA (F statistic). Boldface type indicates statistical significance.

* Data available for 247 patients.

† Data available for 208 patients.

$\ddagger$ Data available for 227 patients.

(Gy), margin dose (Gy), indication for GKRS (residual or recurrent tumor), and treatment of the whole sella, an interval from resection to GKRS of $\leq 3$ months remained associated with a greater probability for endocrine remission at 2 years (HR 1.561,95\% CI 1.033-2.359; $\mathrm{p}=0.034)$ and at last follow-up (HR 1.518, 95\% CI 1.039-2.218; p $=0.031)$. The interval from resection to GKRS of $>12$ months was associated with reduced likelihood of endocrine remission at 2 years (HR 0.637, 95\% CI $0.424-$ $0.957 ; \mathrm{p}=0.030$ ) and at the last follow-up (HR $0.641,95 \%$ 
TABLE 2. Tumor and treatment characteristics as a function of treatment time after surgery in 255 patients with CD

\begin{tabular}{|c|c|c|c|c|c|c|}
\hline \multirow[b]{2}{*}{ Characteristic } & \multirow[b]{2}{*}{ Total Sample } & \multicolumn{4}{|c|}{ Time Interval Between Resection and Radiosurgery } & \multirow[b]{2}{*}{ p Value } \\
\hline & & $\leq 3$ Mos & $4-6$ Mos & $7-12$ Mos & $>12$ Mos & \\
\hline No. of patients & 255 & $77(30 \%)$ & $46(18 \%)$ & $34(13 \%)$ & $98(38 \%)$ & \\
\hline Tumor vol, $\mathrm{cm}^{3}$ & $1.55 \pm 2.00$ & $1.65 \pm 1.68$ & $1.76 \pm 2.48$ & $1.59 \pm 1.74$ & $1.37 \pm 2.10$ & $0.488(0.691)$ \\
\hline Vol treated, $\mathrm{cm}^{3 *}$ & $2.24 \pm 2.21$ & $2.52 \pm 1.65$ & $2.43 \pm 3.14$ & $2.48 \pm 2.33$ & $1.87 \pm 1.94$ & $1.449(0.229)$ \\
\hline Cavernous sinus targeted & $126(49 \%)$ & $52(68 \%)$ & $20(44 \%)$ & $15(44 \%)$ & $39(40 \%)$ & $14.769(<0.002)$ \\
\hline Suprasellar component targeted & $40(16 \%)$ & $14(18 \%)$ & $7(15 \%)$ & $4(12 \%)$ & $15(15 \%)$ & $0.776(0.855)$ \\
\hline Whole sella targeted & $70(28 \%)$ & $19(25 \%)$ & $10(22 \%)$ & $8(24 \%)$ & $33(34 \%)$ & $3.219(0.359)$ \\
\hline Margin dose, Gy & $23.21 \pm 6.02$ & $22.10 \pm 1.17$ & $23.68 \pm 7.01$ & $24.69 \pm 6.54$ & $23.34 \pm 6.49$ & $1.679(0.172)$ \\
\hline Maximal dose, Gy & $45.55 \pm 12.21$ & $46.23 \pm 8.27$ & $45.33 \pm 14.82$ & $47.49 \pm 13.25$ & $44.44 \pm 13.13$ & $0.637(0.592)$ \\
\hline Isodose line, $\%$ & $51.74 \pm 9.10$ & $48.23 \pm 6.16$ & $52.87 \pm 7.93$ & $53.03 \pm 8.74$ & $53.52 \pm 10.86$ & $5.837(<0.001)$ \\
\hline No. of isocenters & $7.43 \pm 5.40$ & $8.59 \pm 4.99$ & $7.43 \pm 5.46$ & $7.91 \pm 6.68$ & $6.37 \pm 5.06$ & $2.573(0.055)$ \\
\hline Maximal dose to optic chiasm, Gy† & $5.67 \pm 2.69$ & $5.16 \pm 2.47$ & $5.67 \pm 2.89$ & $6.07 \pm 2.83$ & $5.97 \pm 2.71$ & $1.446(0.230)$ \\
\hline
\end{tabular}

Values are expressed as the mean \pm SD or the number (\%). The $p$ values (in parentheses) were calculated as follows: categorical data were compared using the Pearson chi-square test, and continuous data using the ANOVA ( $F$ statistic). Boldface type indicates statistical significance.

* Data available for 232 patients.

† Data available for 226 patients.

CI $0.448-0.919 ; \mathrm{p}=0.015)$. However, when the treatment volume included the cavernous sinus, only treatment with GKRS > 12 months after the pituitary adenoma resection remained associated with a lower probability of endocrine remission (HR 0.683, 95\% CI 0.475-0.980; $\mathrm{p}=$ $0.039)$.

CD recurrence was documented in $18 \%$ of patients among the 175 who initially achieved endocrine remission after GKRS. Endocrine recurrence rates were not different as a function of the interval from resection to GKRS.

\section{Imaging Outcomes and Adverse Events}

The mean time from GKRS to the most recent MRI scan was $56.0 \pm 44.1$ months (median 48 months), and this time was balanced across the 4 treatment groups $(\mathrm{p}=$ 0.806; Table 4). In 13 patients (5\%), there was an increase of the adenoma size by at least $20 \%$ during the followup period, whereas the adenoma remained stable or decreased in volume by at least 30\% after GKRS in $98(41 \%)$ and $127(53 \%)$ patients, respectively. Imaging outcomes were not different as a function of time from resection to GKRS.

Incidences of some degree of new pituitary deficiency or new visual and other cranial nerve deficits were not significantly related to time from resection to GKRS (Table 4). The need for additional resection or medical therapy was not related to the interval between resection and GKRS. Twenty patients died during the study period, and the overall mortality rate was similar among the 4 study groups $(\mathrm{p}=0.615)$.

\section{Discussion}

To the best of our knowledge, this is the first study that evaluated the association of the interval between resection and GKRS with treatment outcomes in patients with CD. In this large multiinstitutional series of consecutive patients with CD who underwent GKRS after resection of an ACTH-secreting pituitary adenoma, we found that early GKRS ( $\leq 3$ months after surgery) was associated with greater endocrine remission rates, whereas late GKRS ( $>$ 12 months after surgery) was associated with a reduced probability of endocrine remission. Both early and delayed GKRS had a similar safety profile that was acceptable.

Our most important observation is that in $\mathrm{CD}$ after noncurative resection of an ACTH-producing pituitary adenoma or disease recurrence, early GKRS $(\leq 3$ months of resection) was associated with improved endocrine remission, whereas delayed GKRS (i.e., > 12 months after surgery) was associated with less favorable outcomes. These associations were independent of patients' sociodemographic and clinical characteristics, tissue volume treated, and maximal and margin doses of radiation. Thus, for patients with $\mathrm{CD}$ in whom complete adenoma resection cannot be achieved, with a resulting remission documented by endocrine testing, such patients can benefit from early rather than delayed GKRS. Earlier endocrine remission after SRS has important clinical benefits, including shorter duration of hypercortisolemia and medical treatment and possibly fewer medical treatments, which were implicated as important factors for the long-term prognosis and survival of patients with CD., ${ }^{2,7}$ To the best of our knowledge, there are no studies exploring the optimal timing of GKRS after unsuccessful resection of an ACTH-producing pituitary adenoma. A small study in 7 patients with CD treated with CyberKnife SRS (CKSRS) found that all 4 patients who received CKSRS treatment within 14 months (range 3-14 months) after resection achieved endocrine remission, whereas all 3 patients who were treated with CKSRS more than 14 months (range 15-85 months) after resection had active CD at a follow-up of 55 months. ${ }^{14}$ We studied only those patients who underwent GKRS after an incomplete resection and a failure to achieve remission following surgery, and this approach is consistent with current CD treatment guidelines. ${ }^{15}$ Therefore, early SRS should be considered in pa- 

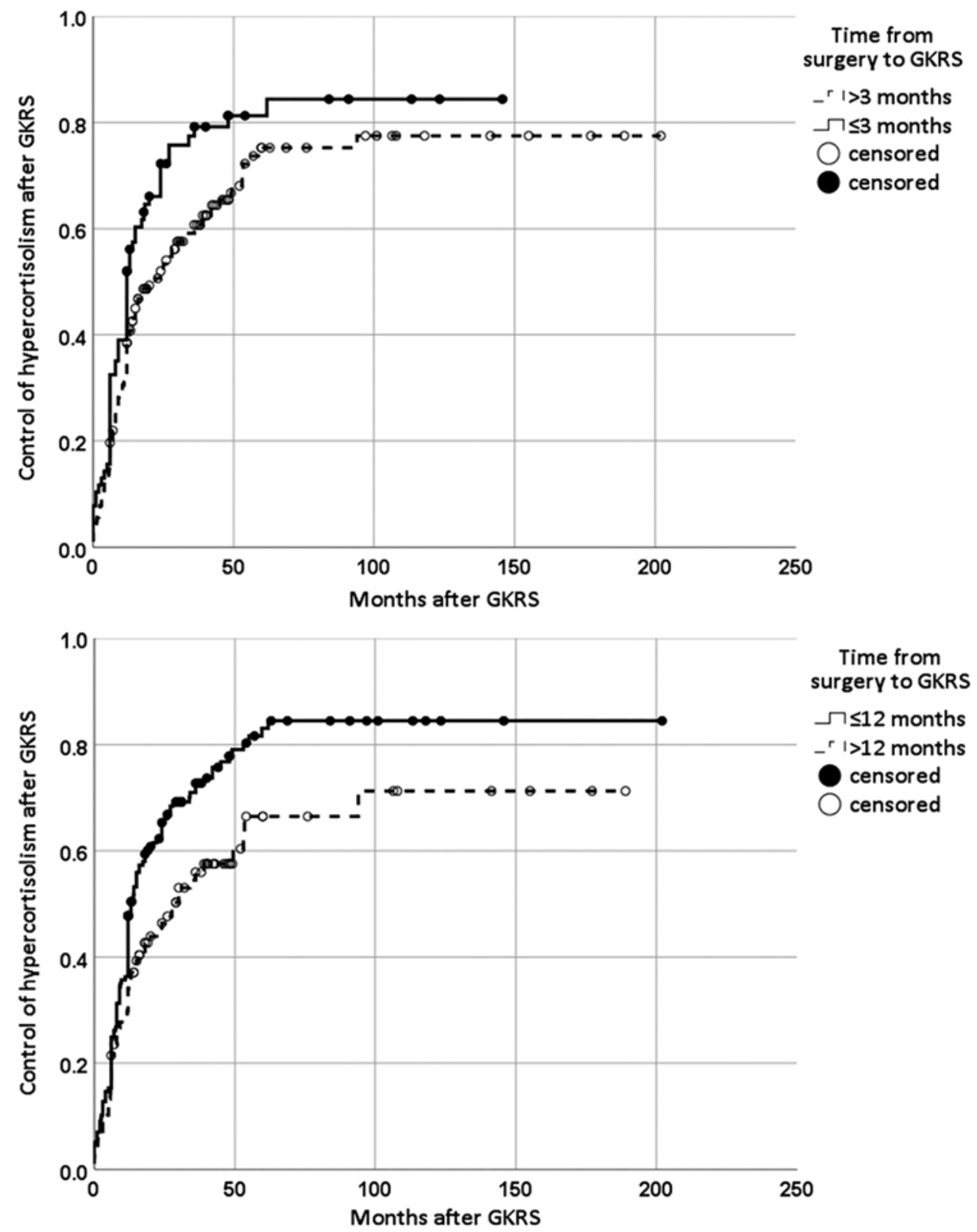

FIG. 1. Kaplan-Meier analyses of the association of time between resection and GKRS to endocrine remission. Upper: Patients dichotomized into groups of $\leq 3$ months and $>3$ months. Lower: Patients dichotomized into groups of $\leq 12$ months and $>12$ months.

tients with $\mathrm{CD}$ after an incomplete resection and persistent $\mathrm{CD}$ after surgery in order to optimize endocrine control and overall prognosis.

The benefits of treating patients with early SRS after resection are likely multifactorial. Early SRS is when the target volume is at its smallest; a smaller target volume has generally been associated with more favorable outcomes following SRS. Also, resection is in essence a controlled trauma at the operative site. Trauma has been associated with the production of tumor necrosis factor, proinflammatory cytokines, and microvascular disruption, and performing a resection even with great care probably leads to at least a temporary cascade of proinflammatory cytokines and microvascular disruption in the sellar region, thereby placing residual pituitary adenoma cells under stress..$^{11,22}$ Delivering SRS early after surgery, when the remaining pituitary adenoma cells are more vulnerable, may induce a higher degree of postradiosurgical inactivation of ACTH production, and this could translate to the improved rate of remission seen with early GKRS in this study.

In our series, actuarial endocrine remission rates ranged from $57 \%$ in the late (>12 months) GKRS group to $78 \%$ in the early ( $\leq 3$ months) GKRS group, and this underscores the clinically meaningful differences of treatment success as a function of time from resection to GKRS. Endocrine recurrence rates were similar among the treatment groups. 
TABLE 3. Association of interval between resection and GKRS with remission in univariate and multivariate Cox regression analyses in 255 patients with $C D$

\begin{tabular}{|c|c|c|c|}
\hline Time From Surgery to GKRS & Univariate & Multivariate* $^{*}$ & Multivariate $†$ \\
\hline \multicolumn{4}{|c|}{ Dependent variable: endocrine remission at 6 mos after GKRS } \\
\hline$\leq 3$ mos after GKRS & $1.686(1.009-2.817), 0.046$ & 0.227 & - \\
\hline 4-6 mos after GKRS & $0.570(0.259-1.254), 0.162$ & - & - \\
\hline 7-12 mos after GKRS & $0.908(0.413-1.998), 0.811$ & - & - \\
\hline$>12$ mos after GKRS & $0.851(0.501-1.447), 0.552$ & - & - \\
\hline \multicolumn{4}{|c|}{ Dependent variable: endocrine remission at 2 yrs after GKRS } \\
\hline$\leq 3$ mos after GKRS & $1.571(1.121-2.204), 0.009$ & 1.561 (1.033-2.359), 0.034 & 0.190 \\
\hline 4-6 mos after GKRS & $0.766(0.490-1.196), 0.241$ & - & - \\
\hline 7-12 mos after GKRS & $1.444(0.924-2.256), 0.107$ & - & - \\
\hline$>12$ mos after GKRS & $0.637(0.446-0.908), 0.013$ & $0.637(0.424-0.957), 0.030$ & 0.115 \\
\hline \multicolumn{4}{|c|}{ Dependent variable: endocrine remission anytime during the follow-up } \\
\hline$\leq 3$ mos after GKRS & 1.450 (1.060-1.984), 0.02 & 1.518 (1.039-2.218), 0.031 & 0.215 \\
\hline 4-6 mos after GKRS & $0.850(0.577-1.253), 0.412$ & - & - \\
\hline 7-12 mos after GKRS & $1.490(0.994-2.234), 0.053$ & - & - \\
\hline$>12$ mos after GKRS & $0.650(0.472-0.893), 0.008$ & $0.641(0.448-0.919), 0.015$ & $0.683(0.475-0.980), 0.039$ \\
\hline
\end{tabular}

- $=$ not done.

Values are expressed as $\mathrm{HR}(95 \% \mathrm{Cl})$, p value. Boldface type indicates statistical significance.

* Adjusted for patient age, sex, pre-GKRS fractionated radiation therapy, medical treatment at time of GKRS, pre-GKRS UFC concentration, volume treated, maximal GKRS dose (Gy), margin dose (Gy), and indication for GKRS (residual or recurrent tumor).

$\uparrow$ Addition of cavernous sinus treatment as predictor variable.

Others have reported endocrine remission rates of $35 \%{ }^{6}$ to $72 \%{ }^{5}$ after GKRS, $57 \%$ after CKSRS,,$^{14} 22 \%{ }^{26}$ to $59 \%{ }^{25}$ after linear accelerator (LINAC)-based SRS, and 54\% after proton beam therapy, ${ }^{24}$ with the median post-SRS follow-up in all studies exceeding 2 years. However, the association of time between resection and SRS with treatment outcomes was reported in only one study. ${ }^{14}$ Findings from the present study indicate that time between resection and SRS therapy should be considered when reporting SRS outcomes of patients with CD.

Management of patients with residual or refractory CD is challenging. ${ }^{1}$ Patients with refractory $\mathrm{CD}$ can be managed with repeated resection, SRS, or medical management, but unfortunately all 3 options provide limited and variable success..$^{15}$ Our study indicates the importance of GKRS for the management of patients with CD after noncurative surgery. A previous study in 20 patients with CD who had disease recurrence after initial SRS found that endocrine remission was achieved in $60 \%$ of patients after a second SRS treatment at a mean follow-up of 6.6 years (range 1.4-19.1 years). ${ }^{12}$ Hence, SRS is important for the management of residual or recurrent $\mathrm{CD}$.

Adenoma growth on neuroimaging was low (5\%) and similar among the 4 treatment groups. High control of growth rates in ACTH-producing pituitary adenomas was previously reported after GKRS,${ }^{5}$ CKSRS,${ }^{14}$ LINACbased systems,${ }^{26}$ and proton beam therapy ${ }^{24}$ Although tumor control after SRS is beneficial for avoiding growth that may lead to cranial nerve and normal pituitary gland dysfunction, the primary goal of SRS management of CD is control of hypercortisolemia in order to prevent longterm metabolic complications and improve prognosis. ${ }^{15}$

\section{Limitations and Strengths of the Study}

This study has limitations. Due to the retrospective study design, we were unable to reliably identify underlying medical reasons that prompted the decision to change from medical management to GKRS. There were differences in prior treatments and tumor characteristics as a function of the interval from surgery to GKRS; however, the majority of them could be systematically accounted for only by performing a randomized controlled trial, which would be challenging for this rare disorder. Volumes of pituitary adenomas were small but different between cohorts of patients with early and late treatment; however, in a larger study with pituitary adenomas in patients with CD treated with SRS, tumor volume within typical radiosurgical targets was not a prognostic factor in endocrine remission. ${ }^{13}$ Also, patients with delayed GKRS had more pre-GKRS fractionated radiation therapy, and this was largely a reflection of earlier treatment approaches and did not result in a significant lowering of the therapeutic GKRS dose to the target in either group. Furthermore, information regarding intraoperative findings in terms of tumor invasion of the cavernous sinus and preoperative imaging data was not always available; thus, we were unable to identify factors that precluded complete adenoma resection. Also, the timing from resection to GKRS was determined by the treating teams and probably varied among centers. Also, because we analyzed patients who were treated at 10 participating centers over 24 years, there have been substantial technological improvements of GKRS systems over time that could introduce variation in GKRS planning and delivery both within and among centers. However, the principles of GKRS planning, treat- 
TABLE 4. Imaging outcomes and adverse events after GKRS in 255 patients with CD

\begin{tabular}{|c|c|c|c|c|c|c|}
\hline \multirow[b]{2}{*}{ Outcome } & \multirow{2}{*}{$\begin{array}{c}\text { Total } \\
\text { Sample }\end{array}$} & \multicolumn{4}{|c|}{ Time Interval Between Resection and Radiosurgery } & \multirow[b]{2}{*}{$\mathrm{p}$ Value } \\
\hline & & $\leq 3 \mathrm{Mos}$ & 4-6 Mos & $7-12$ Mos & $>12$ Mos & \\
\hline No. of patients & 255 & $77(30 \%)$ & $46(18 \%)$ & $34(13 \%)$ & $98(38 \%)$ & \\
\hline Imaging outcomes after GKRS* & & & & & & $10.131(0.340)$ \\
\hline Stable & $98(41 \%)$ & $28(37 \%)$ & $19(43 \%)$ & $15(48 \%)$ & $36(40 \%)$ & \\
\hline $30 \%$ reduction & $127(53 \%)$ & $45(59 \%)$ & $21(48 \%)$ & $13(42 \%)$ & $48(53 \%)$ & \\
\hline $20 \%$ increase & $13(5 \%)$ & $2(3 \%)$ & $2(5 \%)$ & $3(10 \%)$ & $6(7 \%)$ & \\
\hline Tumor not seen before GKRS & $3(1 \%)$ & $1(1 \%)$ & $2(5 \%)$ & $0(0 \%)$ & $0(0 \%)$ & \\
\hline Interval from GKRS to last MRI, mos* & $56.0 \pm 44.1$ & $56.7 \pm 43.0$ & $56.7 \pm 45.2$ & $61.9 \pm 54.2$ & $53.1 \pm 41.0$ & $0.327(0.806)$ \\
\hline \multicolumn{7}{|l|}{ Pituitary deficiency after GKRS } \\
\hline Any & $65(26 \%)$ & $21(27 \%)$ & $10(22 \%)$ & $9(27 \%)$ & $25(26 \%)$ & $0.487(0.922)$ \\
\hline Hypothyroidism & $41(16 \%)$ & $11(14 \%)$ & $7(15 \%)$ & $7(21 \%)$ & $16(16 \%)$ & $0.726(0.867)$ \\
\hline Estrogen/testosterone deficiency & $25(10 \%)$ & $9(12 \%)$ & $4(9 \%)$ & $3(9 \%)$ & $9(9 \%)$ & $0.453(0.929)$ \\
\hline GH deficiency & $27(11 \%)$ & $9(12 \%)$ & $4(9 \%)$ & $7(21 \%)$ & $7(7 \%)$ & $5.093(0.165)$ \\
\hline Diabetes insipidus & $5(2 \%)$ & $2(3 \%)$ & $0(0 \%)$ & $1(3 \%)$ & $2(1 \%)$ & $1.256(0.740)$ \\
\hline Hypocortisolism & $21(8 \%)$ & $11(14 \%)$ & $3(7 \%)$ & $1(3 \%)$ & $6(6 \%)$ & $5.749(0.125)$ \\
\hline New visual deficits after GKRS & $5(2 \%)$ & $2(3 \%)$ & $0(0 \%)$ & $1(3 \%)$ & $2(2 \%)$ & $1.256(0.740)$ \\
\hline New other cranial deficits after GKRS & & & & & & $10.072(0.610)$ \\
\hline CN III & $1(0.4 \%)$ & $0(0 \%)$ & $0(0 \%)$ & $0(0 \%)$ & $1(1 \%)$ & \\
\hline CN V & $1(0.4 \%)$ & $0(0 \%)$ & $0(0 \%)$ & $0(0 \%)$ & $1(1 \%)$ & \\
\hline CN VI & $1(0.4 \%)$ & $0(0 \%)$ & $1(1 \%)$ & $0(0 \%)$ & $0(0 \%)$ & \\
\hline Multiple & $1(0.4 \%)$ & $1(1 \%)$ & $0(0 \%)$ & $0(0 \%)$ & $0(0 \%)$ & \\
\hline Endocrine recurrence $†$ & $32(18 \%)$ & $7(12 \%)$ & $7(23 \%)$ & $7(25 \%)$ & $11(20 \%)$ & $3.056(0.383)$ \\
\hline \multicolumn{7}{|l|}{ Additional treatments after GKRS } \\
\hline Repeat GKRS $\ddagger$ & $32(13 \%)$ & $9(12 \%)$ & $6(13 \%)$ & $5(16 \%)$ & $12(12 \%)$ & $0.332(0.954)$ \\
\hline Pituitary adenoma surgery & $21(8 \%)$ & $8(10 \%)$ & $3(7 \%)$ & $2(6 \%)$ & $8(8 \%)$ & $0.901(0.825)$ \\
\hline Adrenalectomy & $17(7 \%)$ & $7(9 \%)$ & $4(9 \%)$ & $2(6 \%)$ & $4(4 \%)$ & $3.147(0.790)$ \\
\hline Fractionated radiotherapy & $1(1 \%)$ & $0(0 \%)$ & $0(0 \%)$ & $1(0.4 \%)$ & $0(0 \%)$ & $6.467(0.091)$ \\
\hline Initiation of new medical therapy§ & $14(6 \%)$ & $1(1 \%)$ & $5(11 \%)$ & $2(6 \%)$ & $6(6 \%)$ & $5.245(0.155)$ \\
\hline \multicolumn{7}{|c|}{$\begin{array}{l}\mathrm{CN}=\text { cranial nerve. } \\
\text { Values are expressed as the mean } \pm \mathrm{SD} \text { or the number }(\%) \text {. The p values (in parentheses) were calculated as follows: categorical data were } \\
\text { compared using the Pearson chi-square test, and continuous data using the ANOVA (F statistic). } \\
\text { * Data available for } 241 \text { patients. } \\
\dagger \text { In total, } 175 \text { patients initially achieved endocrine remission. } \\
\text { † Data available for } 252 \text { patients. } \\
\text { § Data available for } 251 \text { patients. }\end{array}$} \\
\hline
\end{tabular}

ment volume calculation, and dose selection were homogeneous throughout the course of the study, and a detailed description of GKRS plan data was reported for all patients. Variations of clinical patient management and follow-up strategies both among institutions and within the same institution over time are another potential limitation of this retrospective study. The use of 4 predefined time periods from surgery to GKRS places our results at increased risk for a type I error.

On the other hand, the large sample size of patients with persistent $\mathrm{CD}$, which is a rare but often devastating disease, is a significant strength of this multicenter study, and it allowed us to optimize statistical power and hence fortify the reliability of our findings. Furthermore, all patients included in this report underwent diagnostic and management procedures for $\mathrm{CD}$ (i.e., resection followed by GKRS) as outlined in contemporary CD treatment guidelines,${ }^{15}$ and they were subjected to rigorous biochemical and imaging follow-up-thus underscoring the clinical applicability of our results to contemporary clinical care of patients with persistent $\mathrm{CD}$.

\section{Conclusions}

In patients with $\mathrm{CD}$ who have residual adenoma after resection, early GKRS is associated with improved endocrine remission, whereas delayed GKRS is associated with worse long-term disease control. Early and late GKRS have acceptable and similar safety profiles. Our findings suggest that early (ideally within 3 months, but not later than 12 months) GKRS should be considered in patients with $\mathrm{CD}$ after noncurative resection in order to achieve control of hypercortisolemia and possibly prevent longterm consequences of persistent $\mathrm{CD}$. 


\section{References}

1. Aghi MK: Management of recurrent and refractory Cushing disease. Nat Clin Pract Endocrinol Metab 4:560-568, 2008

2. Clayton RN, Jones PW, Reulen RC, Stewart PM, HassanSmith ZK, Ntali G, et al: Mortality in patients with Cushing's disease more than 10 years after remission: a multicentre, multinational, retrospective cohort study. Lancet Diabetes Endocrinol 4:569-576, 2016

3. Feelders RA, Pulgar SJ, Kempel A, Pereira AM: The burden of Cushing's disease: clinical and health-related quality of life aspects. Eur J Endocrinol 167:311-326, 2012

4. Graversen D, Vestergaard P, Stochholm K, Gravholt CH, Jørgensen JOL: Mortality in Cushing's syndrome: a systematic review and meta-analysis. Eur J Intern Med 23:278-282, 2012

5. Hughes JD, Young WF, Chang AY, Link MJ, Garces YI, Laack NN, et al: Radiosurgical management of patients with persistent or recurrent Cushing disease after prior transsphenoidal surgery: a management algorithm based on a 25-year experience. Neurosurgery [epub ahead of print], 2019

6. Kobayashi T, Kida Y, Mori Y: Gamma knife radiosurgery in the treatment of Cushing disease: long-term results. J Neurosurg 97 (5 Suppl):422-428, 2002

7. Lacroix A, Feelders RA, Stratakis CA, Nieman LK: Cushing's syndrome. Lancet 386:913-927, 2015

8. Lambert JK, Goldberg L, Fayngold S, Kostadinov J, Post KD, Geer EB: Predictors of mortality and long-term outcomes in treated Cushing's disease: a study of 346 patients. J Clin Endocrinol Metab 98:1022-1030, 2013

9. Lindholm J, Juul S, Jørgensen JO, Astrup J, Bjerre P, FeldtRasmussen U, et al: Incidence and late prognosis of Cushing's syndrome: a population-based study. J Clin Endocrinol Metab 86:117-123, 2001

10. Liu JK, Fleseriu M, Delashaw JB Jr, Ciric IS, Couldwell WT: Treatment options for Cushing disease after unsuccessful transsphenoidal surgery. Neurosurg Focus 23(3):E8, 2007

11. Logsdon AF, Lucke-Wold BP, Turner RC, Huber JD, Rosen CL, Simpkins JW: Role of microvascular disruption in brain damage from traumatic brain injury. Compr Physiol 5:11471160,2015

12. Mehta GU, Ding D, Gupta A, Kano H, Sisterson ND, Martinez-Moreno N, et al: Repeat stereotactic radiosurgery for Cushing's disease: outcomes of an international, multicenter study. J Neurooncol 138:519-525, 2018

13. Mehta GU, Ding D, Patibandla MR, Kano H, Sisterson N, $\mathrm{Su} \mathrm{YH}$, et al: Stereotactic radiosurgery for Cushing disease: results of an international, multicenter study. J Clin Endocrinol Metab 102:4284-4291, 2017

14. Moore JM, Sala E, Amorin A, Martinez H Jr, Bhowmik AC, Chang SD, et al: CyberKnife radiosurgery in the multimodal management of patients with Cushing disease. World Neurosurg 112:e425-e430, 2018

15. Nieman LK, Biller BMK, Findling JW, Murad MH, NewellPrice J, Savage MO, et al: Treatment of Cushing's syndrome: an Endocrine Society clinical practice guideline. J Clin Endocrinol Metab 100:2807-2831, 2015

16. Oldfield EH: Cushing's disease: lessons learned from 1500 cases. Neurosurgery 64 (CN_suppl_1):27-36, 2017

17. Patil CG, Veeravagu A, Prevedello DM, Katznelson L, Vance ML, Laws ER Jr: Outcomes after repeat transsphenoidal surgery for recurrent Cushing's disease. Neurosurgery 63:266271, 2008
18. Pendharkar AV, Sussman ES, Ho AL, Hayden Gephart MG, Katznelson L: Cushing's disease: predicting long-term remission after surgical treatment. Neurosurg Focus 38(2):E13, 2015

19. Petersenn S, Beckers A, Ferone D, van der Lely A, Bollerslev J, Boscaro M, et al: Therapy of endocrine disease: outcomes in patients with Cushing's disease undergoing transsphenoidal surgery: systematic review assessing criteria used to define remission and recurrence. Eur J Endocrinol 172:R227R239, 2015

20. Pivonello R, De Leo M, Cozzolino A, Colao A: The treatment of Cushing's disease. Endocr Rev 36:385-486, 2015

21. Sharma ST, Nieman LK, Feelders RA: Comorbidities in Cushing's disease. Pituitary 18:188-194, 2015

22. Simon DW, McGeachy MJ, Bayır H, Clark RSB, Loane DJ, Kochanek PM: The far-reaching scope of neuroinflammation after traumatic brain injury. Nat Rev Neurol 13:171-191, 2017

23. Starke RM, Reames DL, Chen CJ, Laws ER, Jane JA Jr: Endoscopic transsphenoidal surgery for Cushing disease: techniques, outcomes, and predictors of remission. Neurosurgery 72:240-247, 2013

24. Wattson DA, Tanguturi SK, Spiegel DY, Niemierko A, Biller BMK, Nachtigall LB, et al: Outcomes of proton therapy for patients with functional pituitary adenomas. Int J Radiat Oncol Biol Phys 90:532-539, 2014

25. Wein L, Dally M, Bach LA: Stereotactic radiosurgery for treatment of Cushing disease: an Australian experience. Intern Med J 42:1153-1156, 2012

26. Wilson PJ, Williams JR, Smee RI: Cushing's disease: a single centre's experience using the linear accelerator (LINAC) for stereotactic radiosurgery and fractionated stereotactic radiotherapy. J Clin Neurosci 21:100-106, 2014

\section{Disclosures}

Dr. Grills reports stock ownership in and service on the Board of Directors for Greater Michigan Gamma Knife and, through her institution, reports receiving funding for non-study-related research from Elekta. Dr. Kondziolka reports receiving funding for non-study-related research from Brainlab. Dr. Lunsford is a consultant for Insightec and DSMB, and he reports stock ownership in Elekta AB.

\section{Author Contributions}

Conception and design: Sheehan. Acquisition of data: all authors Analysis and interpretation of data: Sheehan, Bunevicius. Drafting the article: Bunevicius, Kano. Critically revising the article: Sheehan, Bunevicius, Kano, CC Lee, Nabeel, El-Shehaby, Abdel Karim, Martinez-Moreno, Mathieu, JYK Lee, Grills, Kondziolka, Martinez-Alvarez, Reda, Liscak, Su, Lunsford, Vance. Reviewed submitted version of manuscript: all authors. Approved the final version of the manuscript on behalf of all authors: Sheehan. Statistical analysis: Bunevicius, Krsek. Administrative/technical/ material support: Sheehan. Study supervision: Sheehan.

\section{Correspondence}

Jason P. Sheehan: University of Virginia Health System, Charlottesville, VA.jps2f@hscmail.mcc.virginia.edu. 